\title{
Effect of Bioconditioning on Surface Characteristics of Periodontally-affected Roots of Diabetic Patients
}

\author{
${ }^{1}$ Khalid M Abdelaziz, ${ }^{2}$ Hossam A Eid, ${ }^{3}$ Refaat A Eid
}

\begin{abstract}
Objective: Advanced glycosylated end products (AGEs) in type II diabetic patients are usually precipitated on the periodontallyaffected root surfaces. The presence of periodontopathic microbes, at the same time, may also add a negative impact on the prognosis of the regenerative periodontal surgery. This in vitro study aimed to evaluate the effect of chemical conditioning on surface characteristics of periodontally-affected roots of diabetic patients.
\end{abstract}

Methods: Three groups $(n=25)$ of freshly-extracted teeth were collected from the outpatient clinics, College of Dentistry, King Khalid University. In group 1, teeth were collected from healthy individuals for orthodontic purpose. Teeth of group 2 were collected from healthy patients with chronic periodontitis, while those of group 3 were collected from diabetic patients with chronic periodontitis. Roots of the collected teeth were examined using the scanning electron microscope (SEM) before and after mechanical surface planning and chemical treatment using normal saline, EDTA gel, Tetracycline HCL (TC) or citric acid (CA) each for 4 minutes ( $n=5$ from each group).

Results: Interpretation of SEM images revealed undesirable etching effect of the chemicals used on the surfaces of healthy roots. Although EDTA showed an effective cleanse of the smear debris, it seemed to have no power on surface cuticles existed on roots of diabetic individuals. Tetracycline $\mathrm{HCl}$ provided acceptable conditioning of periodontally-affected root surfaces. Citric acid showed a powerful removal of both smear debris and cuticle layers off the periodontally-affected roots of diabetic individuals.

Conclusion: Topical application of EDTA, TC or CA shows sensible effect on the periodontally affected root surfaces. However, each of these chemicals exhibits different conditioning power. Citric acid is a promising agent to biomodify the periodontally-affected root surfaces of diabetic patients.

\section{${ }^{1}$ Associate Professor, ${ }^{2,3}$ Assistant Professor}

${ }^{1}$ Dental Biomaterials, Department of Restorative Dental Sciences College of Dentistry, King Khalid University, Abha, Saudi Arabia

${ }^{2}$ Department of Periodontology and Preventive Dental Sciences College of Dentistry, King Khalid University, Abha, Saudi Arabia; Department of Oral Medicine and Periodontology, Faculty of Dentistry, Suez Canal University, Ismailia, Egypt

${ }^{3}$ Scanning Electron Microscope Unit, Department of Medical Pathology, College of Medicine, King Khalid University, Abha Saudi Arabia

Corresponding Author: Khalid M Abdelaziz, Associate Professor Dental Materials, Department of Restorative Dental Sciences College of Dentistry, King Khalid University, Po Box 3263, Abha61741, Saudi Arabia, Phone: +96672418044, Fax: +96672418066 e-mail: bedie001@yahoo.com, kmbder@kku.edu.sa
Keywords: Diabetic patients, Teeth roots, Periodontitis, SEM, Biomodification, Conditioning.

Clinical relevance: Citric acid is an acceptable biomodifier for the periodontally-affected root surfaces. This approach may improve the prognosis of periodontal therapies especially in type II diabetic patients.

How to cite this article: Abdelaziz KM, Eid HA, Eid RA. Effect of Bioconditioning on Surface Characteristics of Periodontally-affected Roots of Diabetic Patients. World J Dent 2014;5(2):81-86.

Source of support: This study is fully-supported by The Scientific and Research Deanship, King Khalid University grant \# 242/1433.

Conflict of interest: None

\section{INTRODUCTION}

Gingival inflammation, detachment and recession and the existence of periodontal pockets are common findings in patients with chronic periodontitis. ${ }^{1}$ Regeneration of the affected periodontal tissues remains a major clinical challenge and is usually complicated in presence of soft and hard deposits on the exposed and subgingival root surfaces. ${ }^{2}$ Several studies indicated that the control of such deposits and the exposure of collagen fibrils usually improve the chances of successful tissue healing. ${ }^{3,4}$ These needs could be achieved using EDTA gels, citric acid (CA), and tetracycline hydrochloride (TC) following thorough scaling and root planning. ${ }^{5-7}$

Gamal and Mailhot $(2003)^{8}$ confirmed the efficiency of EDTA gel root conditioning and reported a significant improvement in periodontal cell adhesion, although dentinal tubules exposure was a noticeable feature accompanying this conditioning approach. ${ }^{9,10}$ In addition to its anti-collagenase activity, TC also seemed a powerful root surface modifier. It showed higher efficiency, in comparison to EDTA, removing the deposited smear layer and uncovering the collagen fibrils. ${ }^{7}$ However, these actions appeared to be more dependent on the morphologic structure of root cementum and the severity of the mechanical instrumentation rather than on the concentration and time of TC application. ${ }^{11}$

Prasad et al $(2012)^{6}$ reported the success of CA in doing the same conditioning function that EDTA and tetracycline do. Others demonstrated higher efficacy of CA and recommended its use for root surface conditioning. ${ }^{9}$ In spite of the reported success of the aforementioned agents on both 
laboratory and animal levels, some authors ${ }^{12}$ claimed that the use of such agent with low $\mathrm{pH}$ may cause necrotizing effect and usually fails to improve the clinical parameters in humans.

On the other hand, type II diabetic patients usually exhibit more severe periodontitis with high plaque index and high prevalence and magnitude of root surface caries. ${ }^{13} \mathrm{~A}$ layer of regular laminar structure, 40 to $80 \mu \mathrm{m}$ thick, named dental cuticle was also noticed to cover the entire length of the periodontally-affected tooth roots of diabetic patients. ${ }^{8}$ The presence of this cuticle may reduce the efficacy of adjunct root conditioning procedure and complicate the regenerative periodontal therapy accordingly. Therefore, this in vitro study aimed to evaluate the effect of three different root conditioning agents, EDTA, TC and CA on surface characters of the periodontally-affected root surfaces of diabetic patients.

\section{MATERIALS AND METHODS}

Seventy five freshly-extracted teeth were collected by two independent contributors from three groups of patients $(\mathrm{n}=20)$; healthy individuals with no periodontitis undergoing orthodontic treatment (G1), healthy individuals with chronic periodontitis (G2), and type-2 diabetic individuals with chronic periodontitis whom following a medical intervention for at least 1 year and showing $\mathrm{HbAlc}$ of 6 to $8 \%$ and no history of other general health problems at the time of their recruitment to this study (G3). Teeth in all groups were generally selected with no carious lesions, no association with infectious or inflammatory lesions or even periodontal intervention for the last 6 months before extraction. The periodontally-affected teeth were specifically selected in company with either gingival recession or periodontal pocket(s) not less than $6 \mathrm{~mm}$ deep, although the existence of extensive mobility (grade III) and poor prognosis were the main reasons of their extraction.

All teeth were decapitated and their root apices were cut off $6 \mathrm{~mm}$ apical to their cement-enamel junction using diamond disks (Edenta AG, AU/SG, Switzerland) running at low speed under copious water irrigation. Each root was also sectioned longitudinally into two halves. The root halves of each group were then randomly classified into five subgroups $(\mathrm{n}=10)$ following the proposed surface conditioning protocol. Root surfaces in SG1 were left with no scaling or root planning and did not receive any kind of chemical conditioning in order to act as basic references. The surfaces of other roots were subjected to ultrasonic scaling (Cavitron Plus, Dentsply, New York, PA) and root planning before their chemical conditioning using normal saline (SG2, control), 17\% EDTA gel (PrepRite, Pulpdent Corp, Watertown, MA) (SG3), aqueous solution of TC (Fisher Scientific, Fair Lawn, NJ) (SG4) and 12.5\% CA (Company Name: Inter-
Med, Inc./Vista Dental Products, Racine, WI) (SG5) for 4 minutes. Cotton pellets damped in CA and TC aided in keeping either chemical in contact with the root surfaces of SG4 and SG5 for the proposed treatment time.

All specimens were thoroughly washed up with saline solution and incubated at $37 \pm 1.2^{\circ} \mathrm{C}$ (Heratherm, Thermo fisher Scientific, Fair Lawn, NJ) for 7 days before assessing their surfaces using the scanning electron microscope (SEM) (JSM6510LV, Jeol, Tokyo, Japan). All specimens were subjected to gold sputter coating and then examined at different magnifications, starting at $\times 50$ through $\times 5000$, to figure out any change acquired as a result of the proceeding root surface conditioning; however, the $\times 100$ magnification was selected to perform the necessary morphometric comparisons. The effectiveness of each conditioning protocol was then graded by 3 evaluators from 1 (the best) to 4 (the worst) depending on its effective removal of surface deposits and following the criteria listed in Table 1. For the statistical purpose, the grade of each specimen was converted into the corresponding point value (see Table 1) before calculating the mean value of each subgroup. Both Kruskal-Wallis test and Mann-Whitney comparisons were used to state the statistical differences between the nonparametrical data of the test subgroups.

\section{RESULTS}

SEM images indicated little or no deposits on healthy root surfaces (Fig. 1), although the images of the periodontallyaffected roots exhibited dense smear deposits in addition to thick cuticle layer in case of those obtained from diabetic patients (Figs 2 and 3). Specimens' inspection under higher magnifications $(\times 5000)$ revealed an evident adhesion of different bacterial strains on surfaces of the periodontallyaffected roots of both healthy and diabetic individuals (Fig. 4).

The application of normal saline helped clean the surfaces of healthy roots following their mechanical debridement. But, at the same time, it had no valuable effect when used against the surface deposits of the periodontallyaffected roots of both healthy and diabetic individuals (Figs 5A to C). On the other hand, the application of other chemical conditioners did change the root surface characteristics. Healthy roots, in most cases, showed a clear etching pattern with obvious exposure of dentinal tubules especially when treated with the CA (Fig. 6). CA looked as a powerful cleansing agent in cases of the periodontallyaffected roots of both healthy and diabetic individuals (Figs 7 and 8), while EDTA, in spite of its effective cleansing of the smear debris (Fig. 9A), it showed a little effect especially on the cuticles existing on the periodontally-affected 


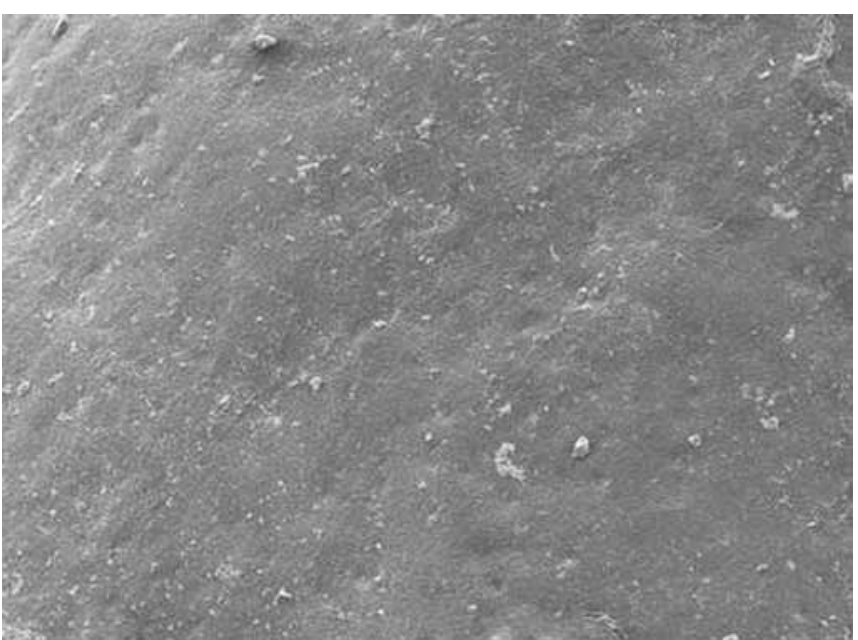

Fig. 1: SEM of healthy root surfaces showing little surface deposits

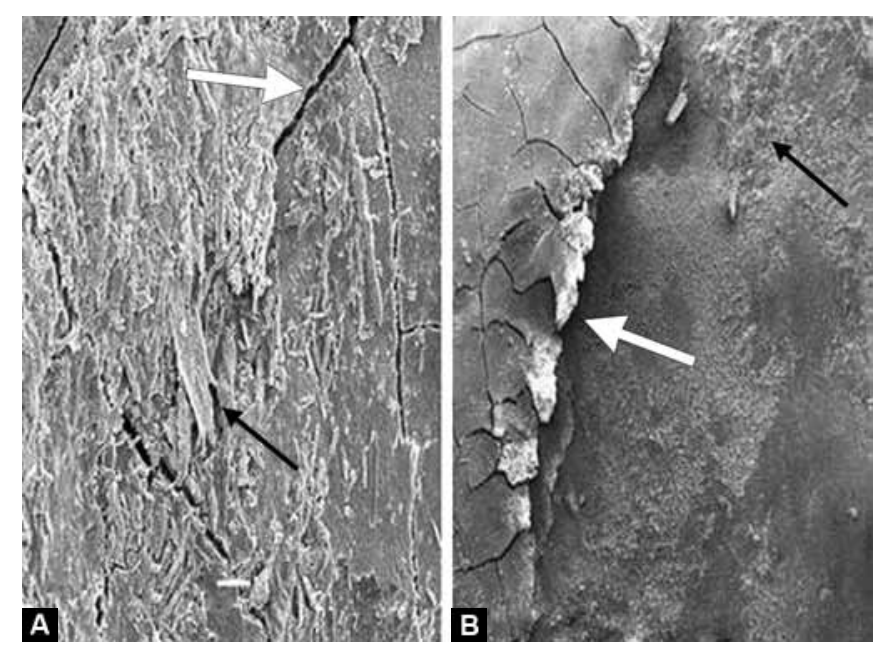

Figs $3 A$ and B: SEM of periodontally-affected root surfaces of diabetic patients showing heavy smear deposits (black arrows) over a thick cuticle layer (white arrows)

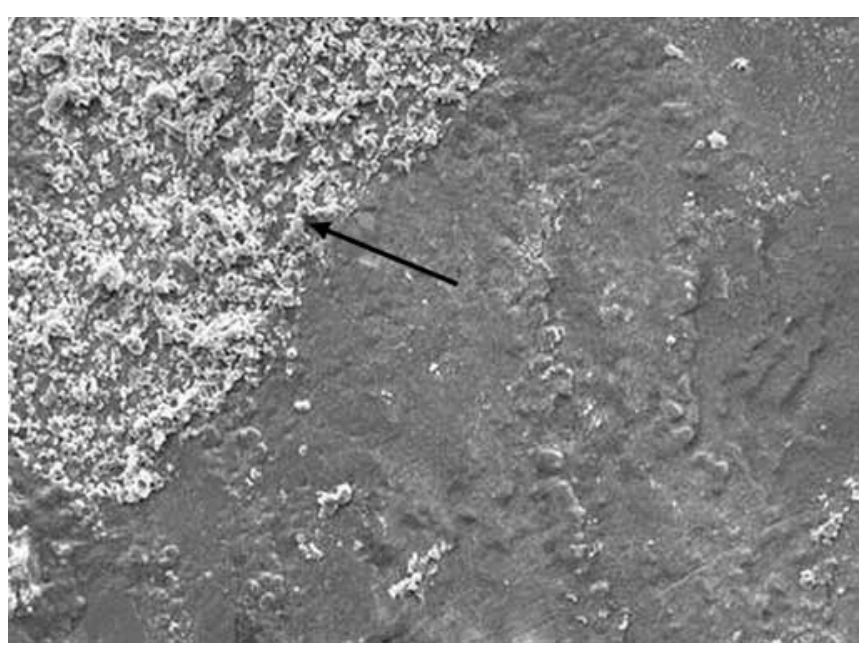

Fig. 2: SEM of periodontally-affected root surfaces patients showing heavy smear deposits (black arrow)

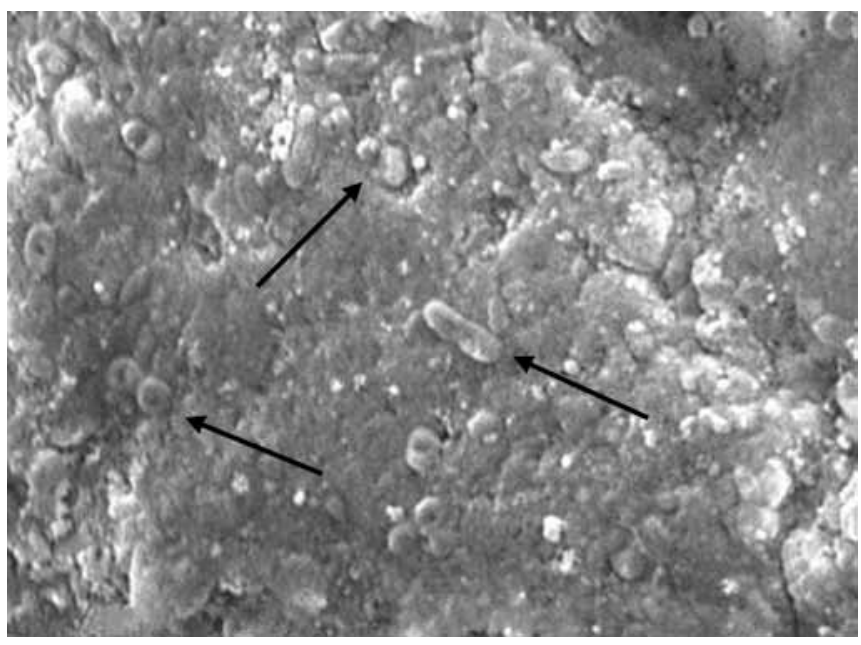

Fig. 4: SEM of periodontally-affected root surfaces showing adhesion of some bacterial strains (black arrows)

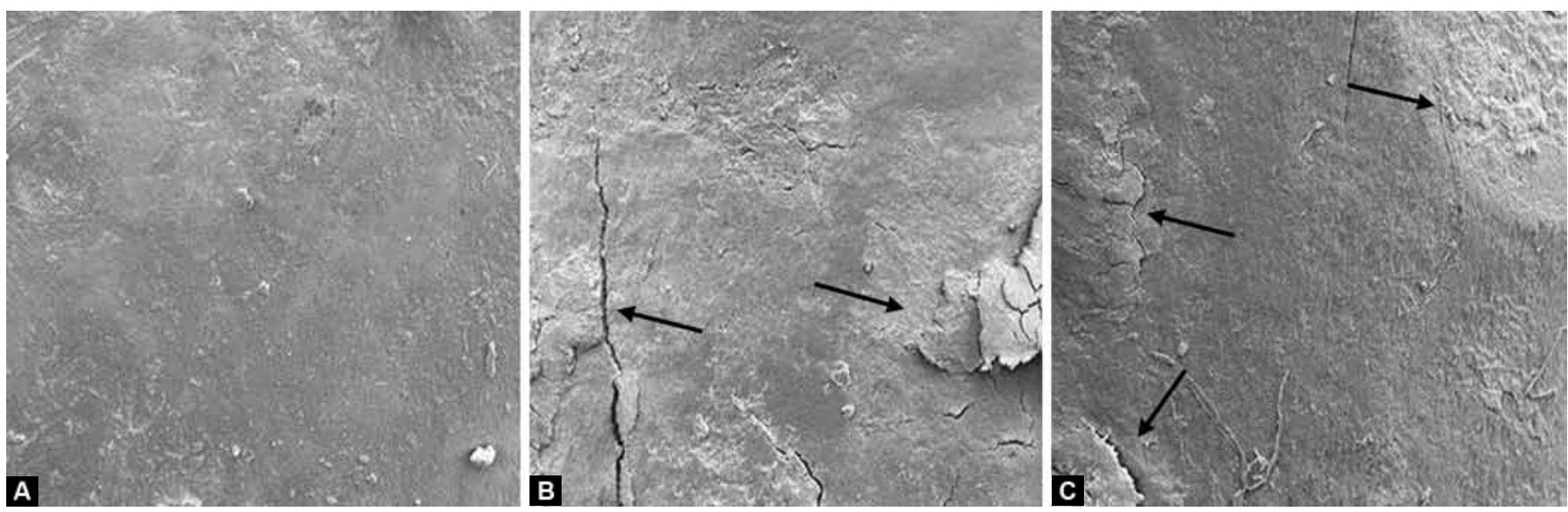

Figs $5 A$ to $C$ : SEM of normal $(A)$ and periodontally-affected root surfaces of healthy $(B)$ and diabetic $(C)$ individuals showing the weak effect of normal saline against surface deposits and cuticle layer (black arrows)

roots of diabetic patients (Fig. 9B). TC had a considerable cleansing action on surface deposits and cuticles; however some of its crystals may remain attached to the root surfaces (Figs 10 and 11).
Table 2 summarizes the results depending on the carried out statistical analysis. The existence of differences between different subgroups was firstly indicated by the KruskalWallis test $(\mathrm{p}=3.81 \mathrm{E}-07)$. The Mann-Whitney comparisons 


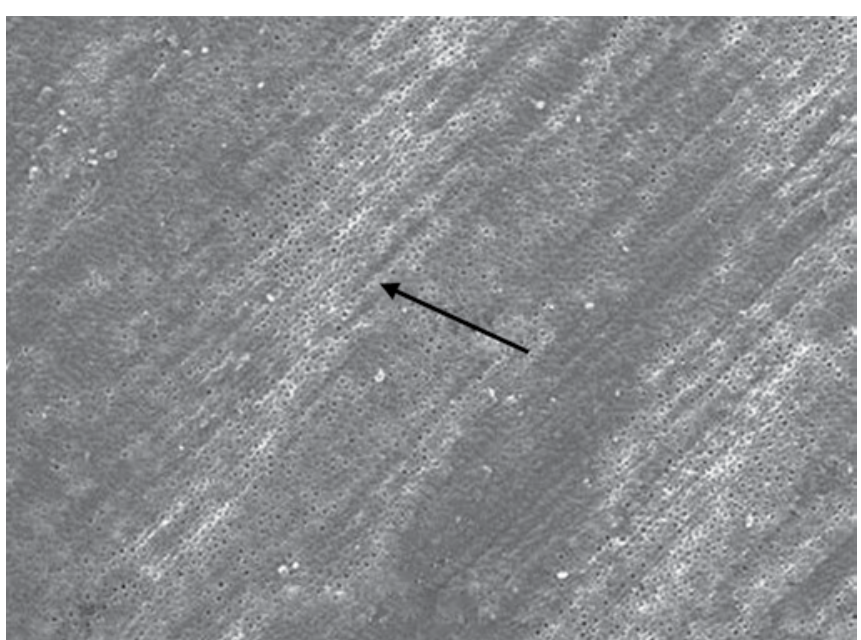

Fig. 6: SEM of citric acid-conditioned healthy root surfaces showing clear etching with obvious exposure of the dentinal tubules (black arrow)

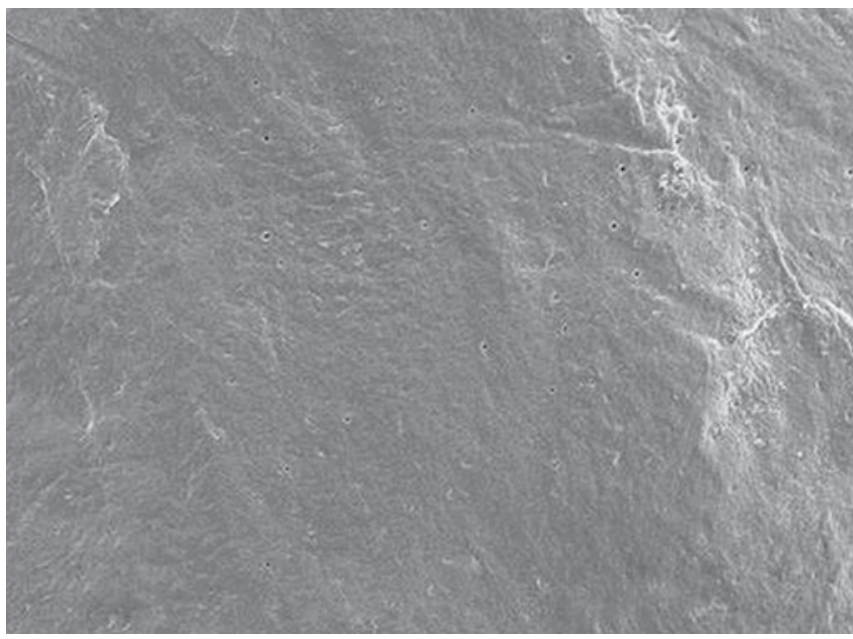

Fig. 8: SEM of citric acid-conditioned periodontally-affected root surfaces of diabetic patients showing powerful cleansing action

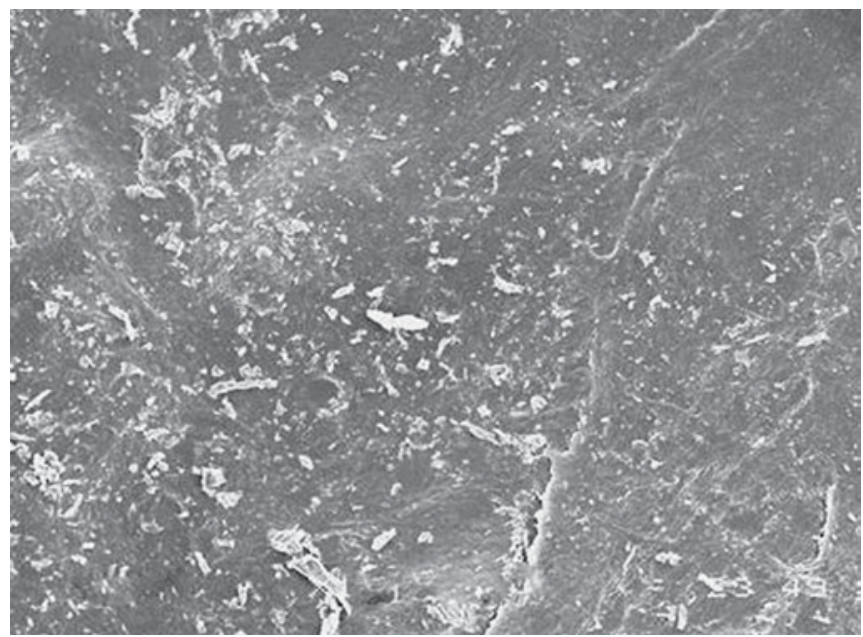

Fig. 10: SEM of tetracycline-conditioned periodontally-affected root surfaces showing acceptable cleansing action

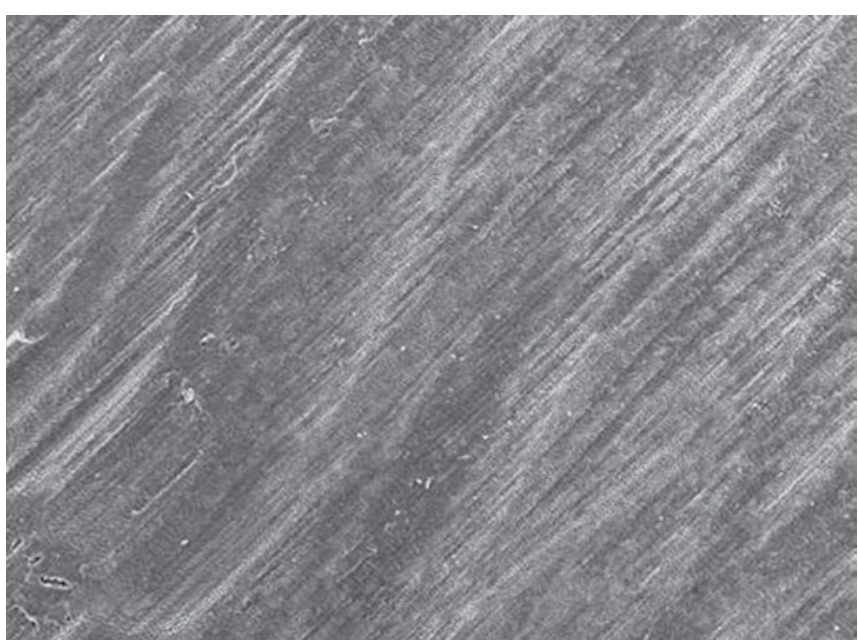

Fig. 7: SEM of citric acid-conditioned periodontally-affected root surfaces showing powerful cleansing action

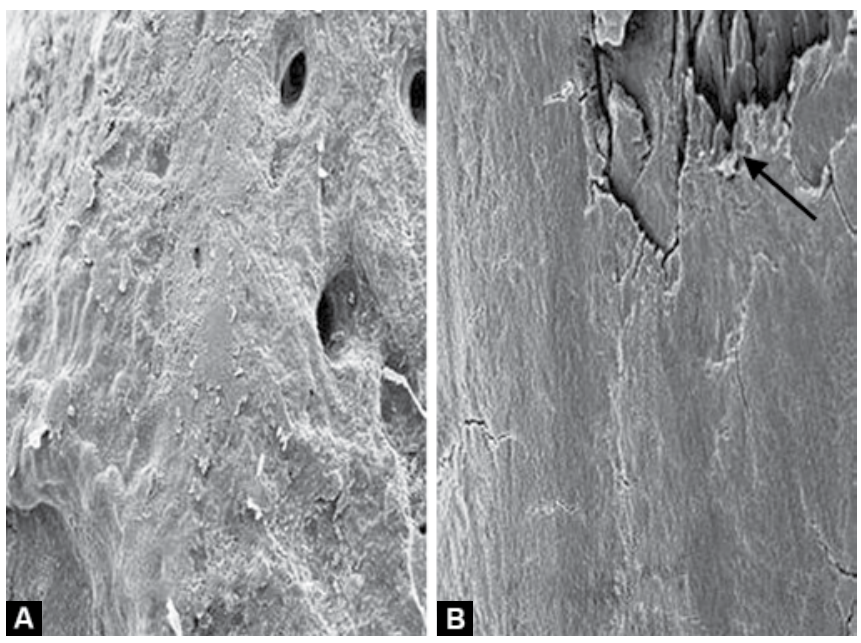

Figs 9A and B: SEM of EDTA-conditioned periodontally-affected root surfaces of healthy $(A)$ and diabetic patients $(B)$ showing powerful effect on smear debris $(A)$ and weak effect on surface cuticles (B) (black arrow)

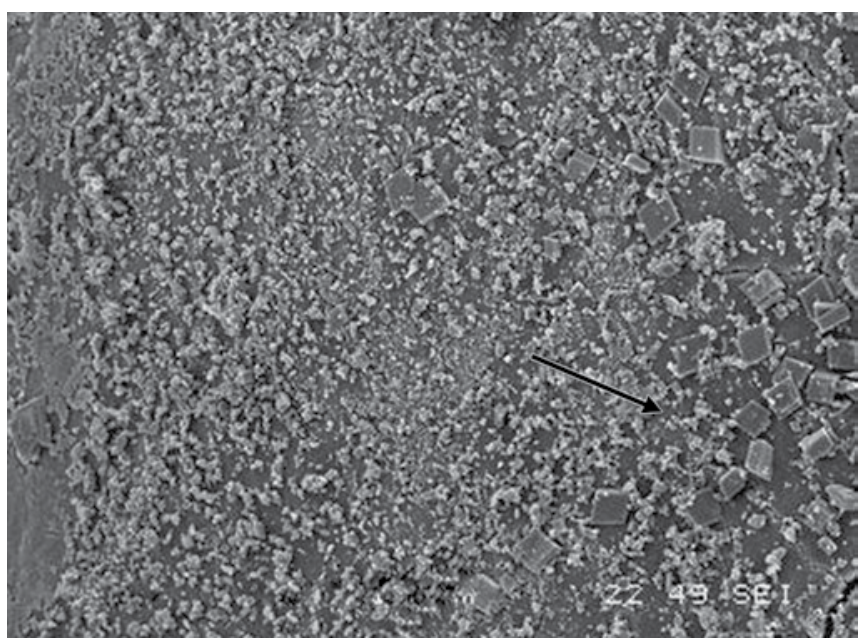

Fig. 11: SEM of tetracycline-conditioned periodontally-affected root surfaces of diabetic patients showing weak cleansing action and crystal precipitate of the tetracycline $\mathrm{HCl}$ (black arrow) 
Table 1: Grading and point value of conditioners' effectiveness

\begin{tabular}{lll}
\hline Grades & Description of SEM finding & $\begin{array}{l}\text { Point } \\
\text { value }\end{array}$ \\
\hline 1 & $\begin{array}{l}\text { Effective removal of surface deposits with no } \\
\text { exposure of dentinal tubules }\end{array}$ & 3 \\
2 & $\begin{array}{l}\text { Complete removal of surfaces deposits with } \\
\text { the exposure of dentinal tubules }\end{array}$ & 2 \\
3 & $\begin{array}{l}\text { Incomplete removal of surface deposits } \\
4\end{array}$ & 1 \\
\hline
\end{tabular}

Table 2: Mean effectiveness value of different chemical conditioners

\begin{tabular}{lllll}
\hline Periodontally-affected & \multicolumn{4}{c}{ Chemical conditioners } \\
\cline { 2 - 5 } root surfaces & Saline & EDTA & TC & $C A$ \\
\hline Healthy individuals & $0.75^{\mathrm{a}}$ & $5.50^{\mathrm{b}}$ & $6.00^{\mathrm{b}}$ & $5.00^{\mathrm{b}}$ \\
Diabetic individuals & $0.50^{\mathrm{c}}$ & $2.00^{\mathrm{d}}$ & $5.25^{\mathrm{b}}$ & $6.50^{\mathrm{e}}$ \\
\hline
\end{tabular}

a,b,c,d,e Significant differences between different test subgroups (Mann-Whitney, $\mathrm{p}<0.05$ ); TC: Tetracycline $\mathrm{HCl}$; CA: Citric acid

confirmed the detected differences between the four tested subgroups of G3 specimens $(p<0.05)$, where CA appeared to have the highest effectiveness against surface deposits and cuticle layer. No statistical differences were confirmed between the effectiveness values of EDTA, TC and CA (SG3, SG4 and SG5) on G2 specimens' surfaces (MannWhitney, $p>0.05$ ), although all of them were significantly powerful than normal saline (SG2, control) when used against specimens' having the same surface characteristics (G2) (Mann-Whitney, p $=0.0044,0.0040$ and 0.0040). In addition, TC showed comparable degrees of effectiveness against G2 and G3 root surfaces (Mann-Whitney, 0.0999).

\section{DISCUSSION}

Gingival attachment and regeneration of the periodontal tissue following either conservative or surgical intervention greatly depend on controlling the local factors existing within the operative area. ${ }^{2}$ The root surfaces of the periodontallyaffected teeth usually show an evident contamination with soft and hard deposits. ${ }^{3,4}$ The existence of type- 2 diabetes may also add a complication toward a successful treatment. Some researchers ${ }^{14}$ reported the formation of a thick cuticle layer on the surfaces of the periodontally-affected roots of diabetic patients. Others ${ }^{3,4}$ deduced the necessity of the mechanical removal of these deposits at the time of managing such cases and believed that the prognosis would be improved following further biomodification of the affected root surfaces. Accordingly, this in vitro study was designed to assess the ability of three different topically-applied chemicals (EDTA, TC and CA), in comparison to normal saline, to condition and biomodify the surfaces of the periodontally-affected teeth of both healthy and diabetic patients.
SEM images proof the presence of smear debris and cuticle coatings (see Figs 2 and 3) on the root surfaces of periodontally-affected teeth of both healthy and diabetic patients. These findings coincide with those of Trombelli et al, (1994) $)^{7}$ and Prasad et al, (2012) ${ }^{6}$ and their existence interferes with the proper healing and attachment of periodontal tissue. ${ }^{2}$ This interference comes through limiting the intimate contact at root-tissue interface. Moreover, the existence of bacterial strains and there products could help the production of collagenase enzyme that destroys the already-formed and the newly-formed collagen attachment. ${ }^{3}$

Topical application of chemical agents on the periodontally-affected root sites is a documented procedure during periodontal therapy, ${ }^{5-7}$ although their use against healthy root tissue could harm the cementum layer and expose the dentinal tubules leading to tooth sensitivity, dental caries and increase the incidence of bacterial migration through those opened tubules toward dental pulp. EDTA is known as a powerful chelating agent and has an often use in endodontics; it helps dissolve the intracanal smear debris and organic precipitates. ${ }^{15}$ Therefore, the use of EDTA over the periodontally-affected root surfaces helps the debridement of the smear precipitates (see Fig. 9A); however, its effectiveness diminished on the cuticle layers of on the root surfaces of diabetics (see Fig. 9B). This could be explained by the uncertainty Walton and Rivera (1996) ${ }^{15}$ about the chelating power of this agent on the organic precipitate.

$\mathrm{TC}$ is famous for its anti-bacterial and anti-collagenase activities. ${ }^{6}$ At the same time, SEM images revealed an evident dissolving effect on the smear debris layer (see Fig. 10) and a sensible effect on roots' surface cuticles (see Fig. 11). Many authors ${ }^{7,16}$ referred these actions to the recognized acidity of TC, although others ${ }^{11,17}$ related TC powerful actions, in comparison to EDTA, to factors other than its concentration and the time of application. In spite of the obvious adhesion of the nondissolved TC crystals to the conditioned root surfaces, probably due to the inadequate solubility of TC at normal water temperature, ${ }^{18}$ the achieved cleaning has been documented to help the normal tissue integration following periodontal intervention. ${ }^{19}$

On the other hand, CA achieved a great success in removing both smear and cuticle deposits off the periodontally-affected root surfaces (see Figs 6 and 7), Labahn et al $(1992)^{20}$ reported higher potency of CA and demonstrated more extensive changes on roots surfaces in comparison to those caused by TC. The excellent results noticed in the specimens treated with AC are mainly referred to its potential acidity and its dissolution and etching power on both organic and inorganic precipitates. ${ }^{17}$ 
Regardless the limitation of this study, the noticed conditioning effect of both TC and CA nominate their routine use to modify surfaces of the periodontally-affected roots of healthy and diabetic individuals. However, further studies are needed to discover the collagenase activity over roots surfaces following their bio-modification using the suggested chemicals.

\section{CONCLUSION}

Topical application of EDTA, TC or CA shows sensible effect on the periodontally-affected root surfaces. However, each of these chemicals exhibits different conditioning power. Citric acid is a promising agent to bio-modify the periodontally-affected root surfaces of diabetic patients.

\section{REFERENCES}

1. Needleman I, Giedrys-Leeper E. British society of periodontology, young practitioners guide to periodontology, Available at: http://www.bsperio.org.uk/publications/downloads/Young Practitioners_Guide.pdf 2012.

2. Gamal AY, Kumper RM. A novel approach to the use of doxycycline-loaded biodegradable membrane and edta root surface etching in chronic periodontitis: a randomized clinical trial. J Periodontol 2012;83:1086-1094.

3. Sander L, Frandsen EV, Arnbjerg D, Warrer K, Karring T. Effect of local metronidazole application on periodontal healing following guided tissue regeneration. Clinical findings. J Periodontol 1994; 65:914-920.

4. Yoshinari N, Tohya T, Kawase H, Matsuoka M, Nakane M, Kawachi M, Mitani A, Koide M, Inagaki K, Fukuda M, et al. Effect of repeated local minocycline administration on periodontal healing following guided tissue regeneration. J Periodontol 2001;72:284-295.

5. Hamilton DW, Oates CJ, Hasanzadeh A, Mittler S. Migration of periodontal ligament fibroblasts on nanometric topographical patterns: influence of filopodia and focal adhesions on contact guidance. PLoS One 2010;5:e15129. e15129. Available from: http://www.plosone.org/article/ info\%3Adoi\%2F10.1371\%2Fjournal.pone.0015129

6. Prasad SS, Radharani C, Varma S, Kumar SV, Sinha S, Bijle MN. Effects of citric acid and EDTA on periodontally involved root surfaces: a SEM study. J Contemp Dent Pract 2012;13:446-451.

7. Trombelli L, Scabbia A, Calura G. Nondiseased cementum and dentin root surface following tetracycline hydrochloride conditioning: SEM study of the effects of solution concentration and application time. Int J Periodontics Restorative Dent 1994; 14:460-469.

8. Gamal AY, Mailhot JM. The effects of EDTA gel conditioning exposure time on periodontitis-affected human root surfaces: surface topography and PDL cell adhesion. J Int Acad Periodontol 2003;5:11-22.

9. Amaral NG, Rezende ML, Hirata F, Rodrigues MG, Sant'ana, AC, Greghi, SL, Passanezi E. Comparison among four commonly used demineralizing agents for root conditioning: a scanning electron microscopy. J Appl Oral Sci 2011;19:469-475.

10. Babay N.Comparative SEM study on the effect of root conditioning with EDTA or tetracycline $\mathrm{HCl}$ on periodontally involved root surfaces. Indian J Dent Res 2000;11:53-57.

11. Ishi EP, Dantas AA, Batista LH, Onofre MA, Sampaio JE. Smear layer removal and collagen fiber exposure using tetracycline hydrochloride conditioning. J Contemp Dent Pract 2008;9:25-33.

12. Kassab M, Cohen RE. The effect of root modification and biomodification on periodontal therapy. Compend Contin Educ Dent 2003;24:31-34.

13. Hintao J, Teanpaisan R, Chongsuvivatwong V, Ratarasan C, Dahlen G. The microbiological profiles of saliva, supragingival and subgingival plaque and dental caries in adults with and without type 2 diabetes mellitus. Oral Microbiol Immunol 2007; 22:175-181.

14. Jian X, Wang C, Wang S. Morphologic characteristics of dental cuticle on teeth affected by diabetic periodontitis. Hua Xi Kou Qiang Yi Xue Za Zhi 1999;17:56-57.

15. Walton RE, Rivera ER. Cleaning and shaping in Walton and Torabinejad, editors. Principals and practice in endodontics. 2nd ed. Philadelphia: Saunders, 1996. p. 214.

16. Stephens CR, Murai K, Brunings KJ, Woodward RB. Acidity constants of the tetracycline antibiotics. J Am Chem Soc 1956; 78:4155-4158.

17. Lafferty TA, Gher ME, Gray JL. Comparative SEM study on the effect of acid etching with tetracycline $\mathrm{HCl}$ or citric acid on instrumented periodontally-involved human root surfaces. J Periodontol 1993;64:689-693.

18. Tetracycline hydrochloride, product information sheet. 2003. Available from: http://www.sigmaaldrich.com/etc/medialib/ docs/Sigma/Product_Information_Sheet/1/t3383pis.Par.0001. File.tmp/t3383pis.pdf.

19. Liu M, Kurihara H. Histologic comparison of treating root surfaces with different acids. Hua Xi Kou Qiang Yi Xue Za Zhi 1999; 17:230-232.

20. Labahn R, Fahrenbach WH, Clark SM, Lie T, Adams DF. Root dentin morphology after different modes of citric acid and tetracycline hydrochloride conditioning. J Periodontol 1992; 63:303-309. 